\title{
Preliminary Study of JPSS-1/NOAA-20 VIIRS Day-Night Band Straylight Characterization and Correction Methods
}

\author{
H. Chen ${ }^{1}$, H. Oudrari ${ }^{1}$, C. Sun ${ }^{2}$, T. Schwarting ${ }^{2}$ and X. Xiong ${ }^{3}$ \\ ${ }^{1}$ Systems \& Applications, Inc., 10210 Greenbelt Rd, Lanham, MD 20706; \\ ${ }^{2}$ Global Science Technology, Inc., 7855 Walker Dr, Greenbelt, MD 20770; \\ ${ }^{3}$ Science Systems and Exploration Directorate, NASA/GSFC, Greenbelt, MD 20771
}

\begin{abstract}
The JPSS-1 (now named NOAA-20) VIIRS instrument has successfully operated since its launch in November 18, 2017. A panchromatic channel onboard NOAA-20 VIIRS is called the day-night band (DNB). With its large dynamic range and high sensitivity, the DNB detectors can make observations during both daytime and nighttime. However, the DNB night image quality is affected by the straylight contamination. In this study, we focused on Earth view data in the midto-high latitude of the northern and southern hemispheres when spacecraft is crossing the day/night terminators at the beginning of NOAA-20 mission. Based on on-orbit data analysis from previous VIIRS sensor onboard S-NPP mission, straylight contamination mainly depends on the Earth-Sun-spacecraft geometry, and it is also detector and scan-angle dependent. Inter-comparison investigation of straylight behavior in both SNPP and NOAA-20 instruments will be conducted to better understand straylight characteristics. The preliminary study has been performed in this paper to mitigate straylight contamination for NOAA-20VIIRS DNB night images. The effectiveness of the straylight correction algorithm, directly adapted from the S-NPP DNB, is assessed for night images in the day/night terminators. Further work has been identified to improve current straylight correction methodology and DNB-based environmental data products.NOAA-20.
\end{abstract}

Keywords: VIIRS, Day-Night Band, DNB, JPSS-1, NOAA-20, Straylight, Correction Calibration

\section{INTRODUCTION}

The Visible Infrared Imaging Radiometer Suite (VIIRS) on-board the first Joint Polar Satellite System (JPSS-1) spacecraft was launched on November 18, 2017. This spacecraft has been renamed NOAA N20 right after launch. Twenty-five days after launched into Earth orbit, NOAA-20 sent back its first VIIRS science data on December 13, 2017 (nadir door open), as part of a series of instrument activation and checkouts that is taking place before the satellite operations transition into operational mode. VIIRS is one of the key instruments onboard JPSS-1 that will improve dayto-day weather forecasting and environmental monitoring. Since its nadir door opened, Earth View (EV) data have been collected for all the reflective solar bands (RSB). The thermal emissive bands (TEB) became operational after January 4, 2018 when the passive cryo-cooler door was opened. The VIIRS observations are made in 22 spectral bands, including 16 moderate resolution bands, 5 imaging bands, and one panchromatic Day-Night band (DNB), with wavelengths ranging from 0.41 to $12 \mu \mathrm{m}$. The calibrated and geo-located radiances and reflectance/brightness-temperature are called Sensor Data Records (SDRs), which are used to generate the Environmental Data Records (EDRs) in support of divers science disciplines and applications.

VIIRS DNB is a panchromatic visible/near-infrared (Vis/NIR) band designed to detect radiance from the brightest daytime scenes down to very dim nighttime scenes illuminated by a quarter moon. Its dynamic range spans over 7 orders of magnitude. It has a wide spectral bandpass, centered at $0.7 \mu \mathrm{m}$. The DNB sensor is a temperature-controlled chargecoupled device (CCD). The CCD focal plane array (FPA) is sectioned into 4 stages, where each stage essentially 
functions as a separate imager, though they all reside on the same FPA. There are total 32 aggregation modes used over the full swath to keep an almost constant ground spatial resolution in all scans. However, the NOAA-20 uses only 22 aggregation modes to compensate for DNB radiometric non-linearity observed at last 11 aggregation modes, 22-32 [11].

The VIIRS senor includes many design features to eliminate its sensitivity to straylight including sources originating from direct solar or Earth illumination. The nadir aperture doors are angled to eliminate the scatter light from the Sun to the scan cavity for all orbits. The rotate telescope assembly (RTA) includes an integral baffle coated with a very low reflectance black paint that rejects scattered light using geometric blockages [1]. The optical system includes two intermediate image planes of RTA and four mirror anastigmatic (FMA), where aperture stops can be employed to limit the transmission of off-axis straylight. However, even with all of advanced design considerations taken into account, there will still be brief times near the vernal equinox when sunlight can impinge directly on the primary mirror, as this effect is observed near twilight terminator orbits in every orbit not only of JPSS-1 DNB, but also SNPP DNB.

Straylight contamination is the most significant cause of quality degradation in DNB nighttime image quality. The contamination is mainly in the mid-to-high latitude regions where the spacecraft is crossing the northern and southern day/night terminators [2-4]. In these regions, the NOAA-20 spacecraft is no longer in the Earth's shadow, and light could enter through the VIIRS Earth view port or solar diffuser screen and scatter within the instrument and make its way to the DNB arrays, providing a significant signal relative to the dark Earth scenes being imaged. The amount of straylight seen by the DNB can be estimated based on EV measurements of the contaminated regions during moonless nights. This analysis is based on the assumption that small signals observed over the dark Earth scene during a new moon are only due to straylight and airglow, once man-made light sources (nightlights) have been removed. The straylight is estimated in this way once per month during the new moon, and the estimate is subtracted from DNB images during calibration. The straylight features are expected to change from month to month on a nearly repeatable yearly cycle, presumably due to the changes in the straylight optical path within the instrument as the Earth-Sunspacecraft geometry changes. In addition, NOAA-20 shows a unique and large straylight feature at the right edge of scan in all DNB night images. his large straylight feature affects NOAA-20 DNB because it is using a special aggregation mode (Option 21) that extended the EV further to the right edge of scan, while SNPP DNB does not use Option 21 (no extended view).

Correction of straylight in DNB images suffers from the low frequency of calibration data: the baseline straylight contamination can only be determined once per month during the new moon. When applying a correction to incoming DNB images, the simplest approach is to apply the straylight correction determined from the most recent new moon. In this paper, we present preliminary results by adapting the S-NPP DNB straylight correction methods. The layout is as follows. Section 2 describes the DNB straylight feature and estimation algorithm, including the straylight correction strategy using the new Moon. Straylight impacts in both the S-NPP and the NOAA-20 have been compared and evaluated in the radiance magnitudes. Section 3 discusses straylight changes before and after the activity of cryo-cooler door opening. Section 4 shows some straylight correction results in the northern and southern hemispheres. Further work needs be done to deal with a special large straylight feature seen at the edge of scan for NOAA-20 DNB. Section 5, we discuss our conclusions.

\section{DNB STRAYLIGHT FEATURE AND ESTIMATION}

The VIIRS DNB straylight contamination is mainly in the mid-to-high latitude regions [1,2]. This impact is confirmed by DNB night images in both NOAA-20 and S-NPP spacecrafts. In general, both northern and southern hemispheres have a one-tune pattern, where straylight enters through EV. However, the straylight feature may be different between two hemispheres. The southern hemisphere can have a two-tune pattern: one is through EV, and the other through SD screen. However, this is only possible when spacecraft is near solar calibration events in the southern day-night terminator crossing. As the two-tune pattern is more sensitive to the spacecraft azimuth angle, it is hard to remove. Magnitudes of the straylight contamination are geography dependent. Figure 1shows two straylight contaminated DNB night images of S-NPP and NOAA-20 from the left to the right, respectively. These two images are located at a similar geolocation site (northern hemisphere) in the same day. The GMT time of S-NPP DNB image (left) is 06:56:35am, July 13, 2018, and that of NOAA-20 DNB image (right) is 07:44:57am, July 13, 2018. Obviously, the upper side of the S- 
NPP image is completely distorted by straylight. However, the NOAA-20 image shows a blurred area, which is still viewable. Note that the right edge of scan in NOAA-20 DNB image has a unique feature, where the straylight is dominated in the pixel range of 3900 to 4064 . This edge straylight is occurred at the same time as the normal straylight contaminated images. The cause(s) of this strong straylight feature is currently unknown.
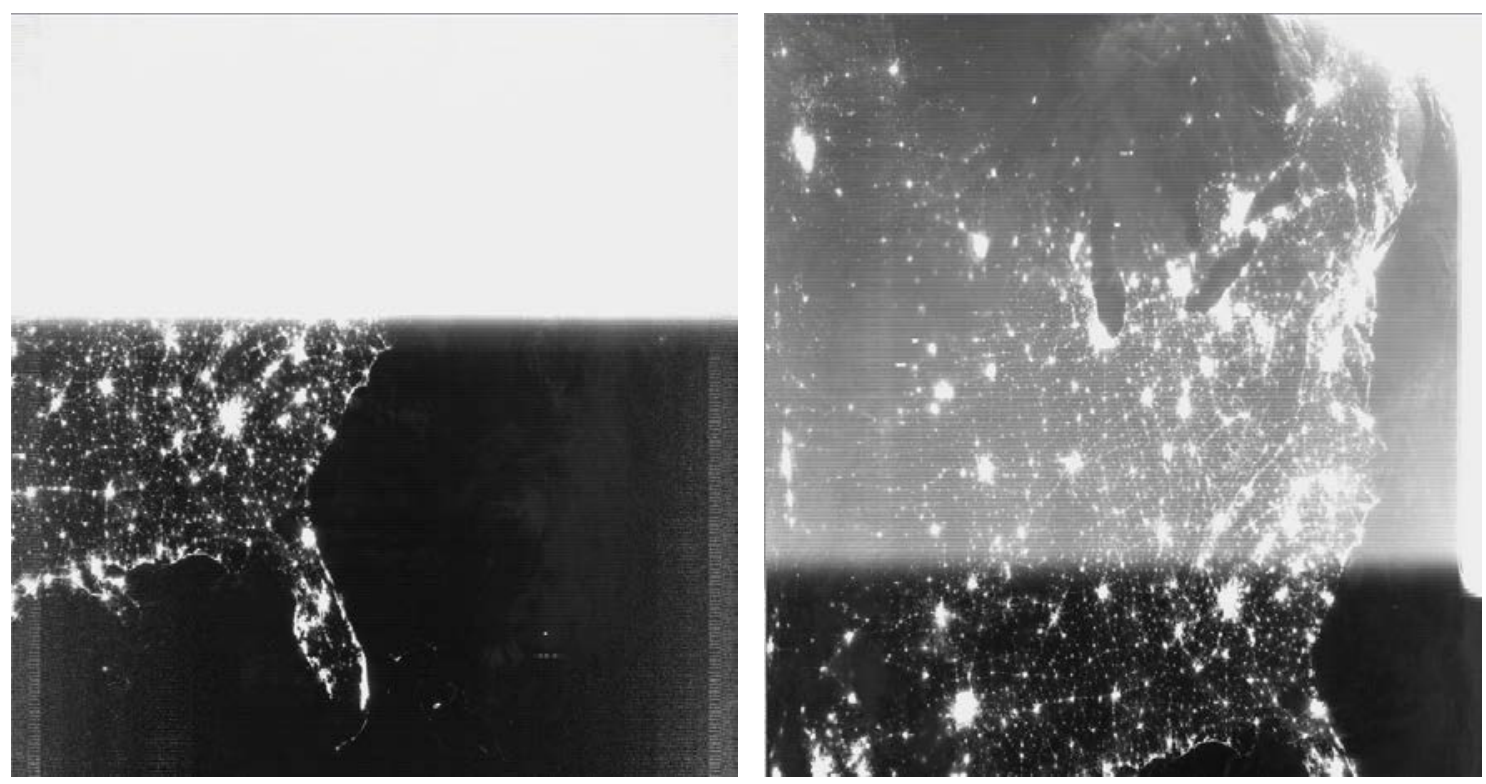

Figure 1 VIIRS DNB night images with straylight contamination: (left) S-NPP DNB image at 06:56:35am, July 13, 2018; (right) NOAA-20 DNB image at 07:44:57am, July 13, 2018.
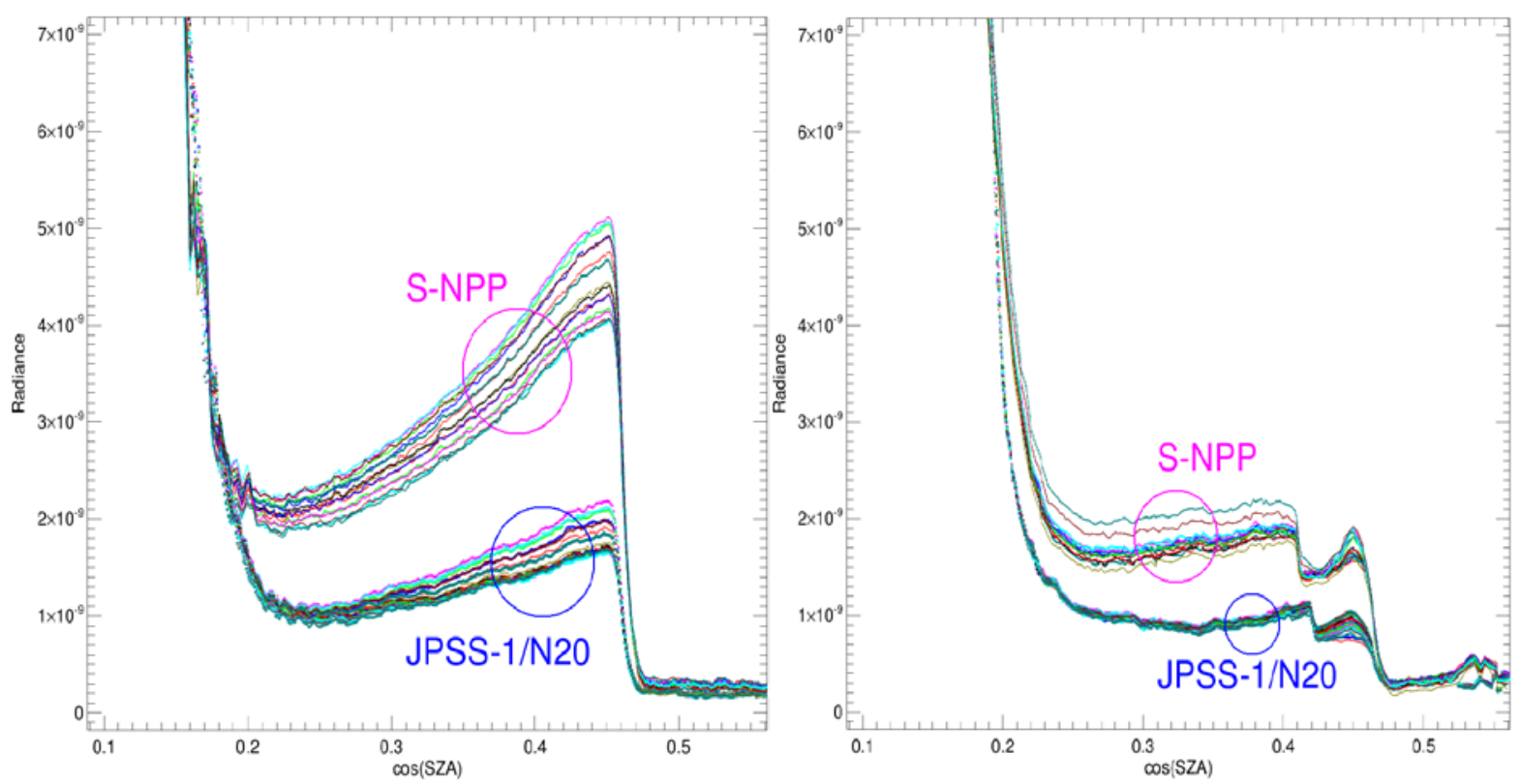

Figure 2 NOAA-20 and SNPP DNB dark scene radiance versus the cosine function of the solar zenith angles on July 13, 2018. Both the northern hemisphere (left) and the southern hemisphere (right) are plotted at the center sample index 2032.

Figure 2 plots the EV dark scene radiance versus the cosine function of the solar zenith angles in the same day. The left chart in Figure 2 shows the EV dark scene radiance in the northern hemisphere computed by data collected on July 13, 
2018. All detectors are plotted with different symbols and/or colors. In this chart, both the S-NPP and NOAA-20 at the center sample index 2032, and with half-angle mirror side 1 (HAM 1) are selected. Between the range of 0.18 to 0.45 in the cosine function of solar zenith angles, similar straylight curves have been observed for all detectors within some magnitude differences in the two groups of S-NPP and NOAA-20, respectively. At the same day, the right chart in Figure 2 shows the EV dark scene radiance in the southern hemisphere, the center sample index 2032 and HAM 1 are selected as well. Notice that there is a second jump, where the cosine function of SZA equals about 0.41 . The straylight contamination range 0.22 0.45 in the cosine function of solar zenith angles. Again, all detectors have a similar behavior, and a two-tune pattern is clearly shown. Over all, the VIIRS DNB straylight contaminations features have a similar shape. However, the NOAA-20 DNB straylight is much smaller than S-NPP in general, roughly from half to one-third radiance difference. In addition, one can observe from Figure 2 that the S-NPP DNB detector difference is much larger than NOAA-20, especially in the southern hemisphere image. The large detector difference will generally show significant strips in DNB night images.

Similar to the case of S-NPP, the algorithm for calculating the NOAA-20 DNB straylight contamination is conducted from the new moon events, and then applying a correction to generate calibrated DNB data. Since the straylight contamination is known to be a function of the Earth-Sun-spacecraft geometry, a straylight correction LUT is defined as a function of SZA, scan angle, and detector. It is generated each month based on the data taken during a new moon. The LUT is used to remove the straylight in regions covering high latitudes of the northern and southern hemispheres. The VCST method used for estimating straylight is similar to other correction methods [2,7] with the same basic assumption that the signal observed over the dark earth surface during a new moon is the result of straylight and airglow. Specifically, there are about 14 orbits of EV data covering the terminator crossings during a new Moon when the Moon and the Sun have the same ecliptic longitude, and the Moon is invisible from the Earth. First, we separate the EV scene signals into 127 bins of 32 pixels in each bin (127x32=4064 is the number of samples in one EV scan). Then, we calculate the radiance of each bin as the mean of lowest $20 \%$ of pixels within the bin. The reason to use the lowest $20 \%$ of pixels is to exclude signal outliers, such as man-made lights, which appear in EV images at a different scan angle each orbit. This is in contrast to the method used by NOAA IDPS, which uses an Earth nighttime light map to exclude nightlights. We take the median of all orbits as the corresponding bin radiance [7-9].

\section{BEFORE AND AFTER CRYORADIATOR DOOR OPEN}

The VIIRS DNB is connected to the warm stage of a cooler, consisting of the cryo-radiator and other passive cooling elements. This cooling system supports the sensor's infrared focal plane arrays, which are responsible for gathering imaging data. Once on orbit, the cryo-radiator will be heated to a temperature of $270 \mathrm{~K}$ to drive off any contaminants from the instrument. After the cryo-cooler door opening, the cold focal planes and the DNB are steadily cooled to operating temperatures, and then stabilized. The NOAA-20 VIIRS cryo-radiator door was opened on January 3, 2018. The dark offsets, LGS gains and gain ratios reached stable levels for all DNB detectors modes and two ham sides around January 4, 2018. However, straylight contamination on DNB night image seems to have increased after cryo-radiator door opening. To illustrate this phenomena, an example is used to compare straylight magnitude before and after VIIRS cryo-door opening. 

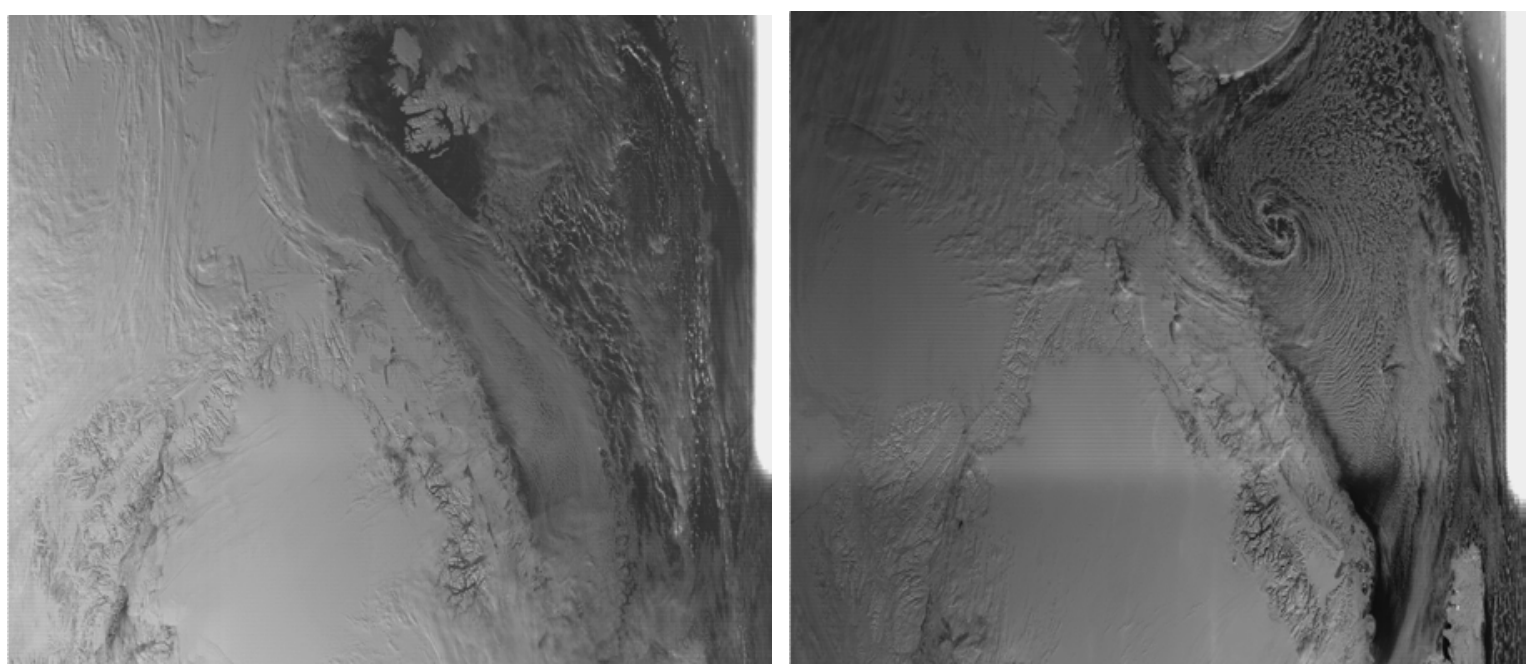

Figure 3 NOAA-20 DNB images: (left) 06:11:33am 01/01/2018; (right) 06:36:52am, 01/05/2018.

Figure 3 shows two NOAA-20 DNB night images, one is on January 1, 2018, before cryo-door open, and the other is on January 5, 2018, after cryo-door open. They are in a half hour time range, and a similar geo-location around $(80,135)$, the latitude and the longitude, respectively. There are total 144 scans and each scan having 4064 pixels plotted. As the VIIRS instrument is in large temperature difference before and after cryo-door open, the DNB calibration is independently performed in these two situations. Before cryo-door open, VROP data is used for dark offset estimations. It is necessary to mention that the DNB calibration is more accurate after cryo-door open, as the VIIRS is in nominal operations (cooled DNB CCD). Visually, we can see larger contrast between the center and the bottom parts in the right image. Notice that both images have a bright white area on their right side, which are corresponding to pixel indices 3900-4064. So far, the reason of this edge of scan large straylight contamination is unknown. However, we may use this special area to illustrate straylight contamination changes before and after the cryo-door open event. 

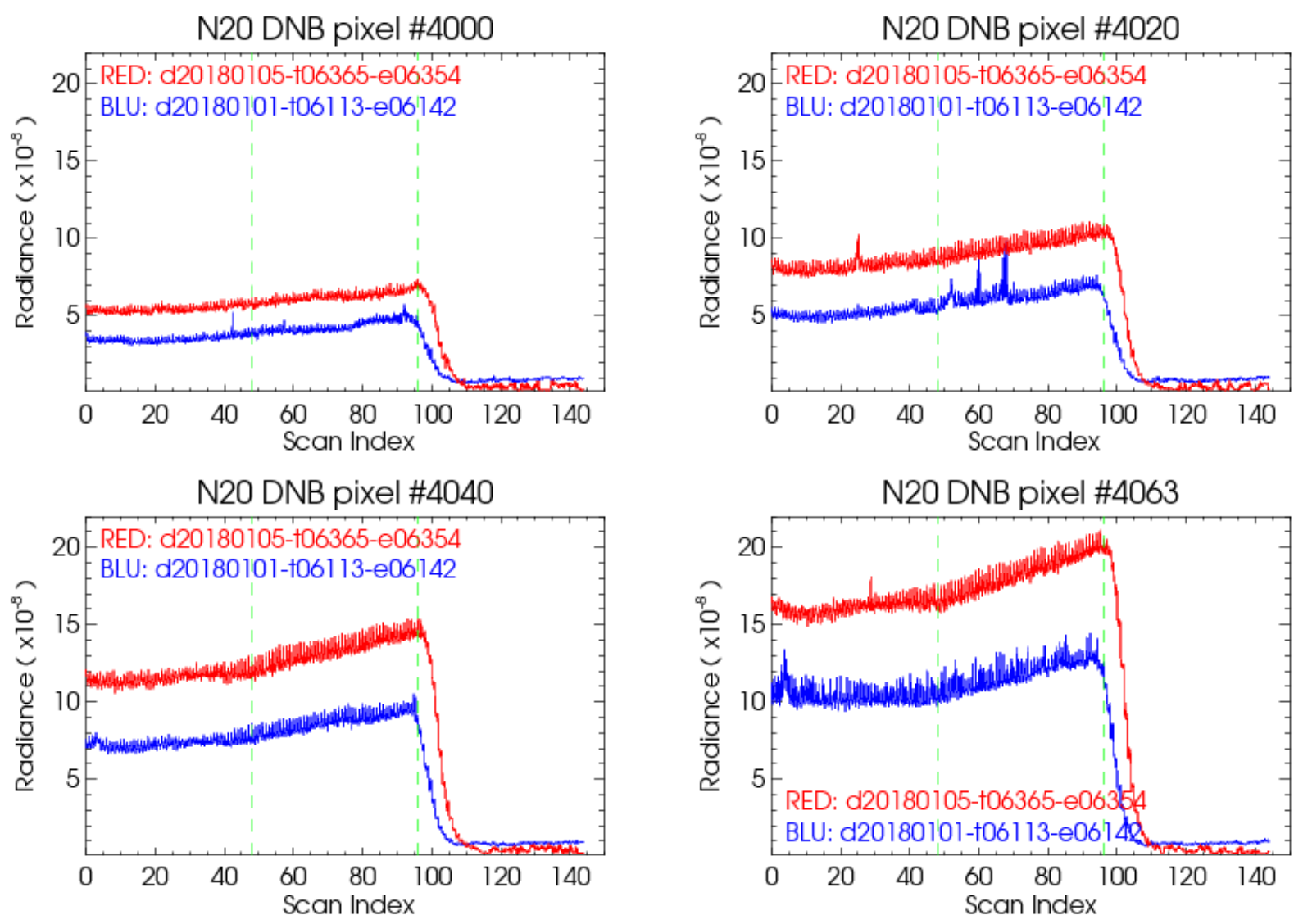

Figure 4 DNB radiance from a vertical transect at four sample indices, 4000, 4020, 4040 and 4063.

Figure 4 plots four-pixel-index radiance trends of the two DNB images in Figure 3. The edge samples of 4000, 4020, 4040, 4063 indices are used for 144 scans for all 16 detectors. Two vertical transects in each sub-plots are used to separate three granules in 48 scans each. Scans larger than 110 may be treated as normal night images with airglows. Based on radiance charts in Figure 4, it is obviously that day-20180105 has much larger straylight contaminations than day-20180101 in these special edge of scan area seen in these DNB night images. Note that scans of normal night image (larger 110), the case of after-cryo-door-open provides more accurate calibration results as expected. If considering radiance in the case of before-cryo-door-open normalized to the other case, we can get even much smaller straylight contaminations before 100 scans in the examples considered.

\section{STRAYLIGHT CORRECTION PERFORMANCE}

The NOAA-20 DNB straylight estimation methods have been adapted from the S-NPP, and straylight correction LUTs have been developed for mission-wide during Level 1B (L1B)/SDR reprocessing. Both NASA VCST and NOAA IDPS use the same principles for developing the DNB straylight correction algorithm. In this section, we will select two examples of the northern and the southern hemispheres to demonstrate the effectiveness of NOAA-20 DNB straylight correction.

Figure 5 shows the straylight correction result of the example NOAA-20 DNB image over the North America at 07:44:57am, July 13, 2018. The straylight affected nighttime scene is originally plotted in the right of Figure 1 . It is necessary to mention that for all night images in this paper, the radiance scale is $\left[0,3 \times 10^{-9}\right] \mathrm{W} \cdot \mathrm{cm}^{-2} \cdot \mathrm{sr}^{-1}$. After correction, 
most of the straylight contamination is removed, except at the edge of scan area, where the current correction is not as good as expected. It is our next task to deal with this special contamination.

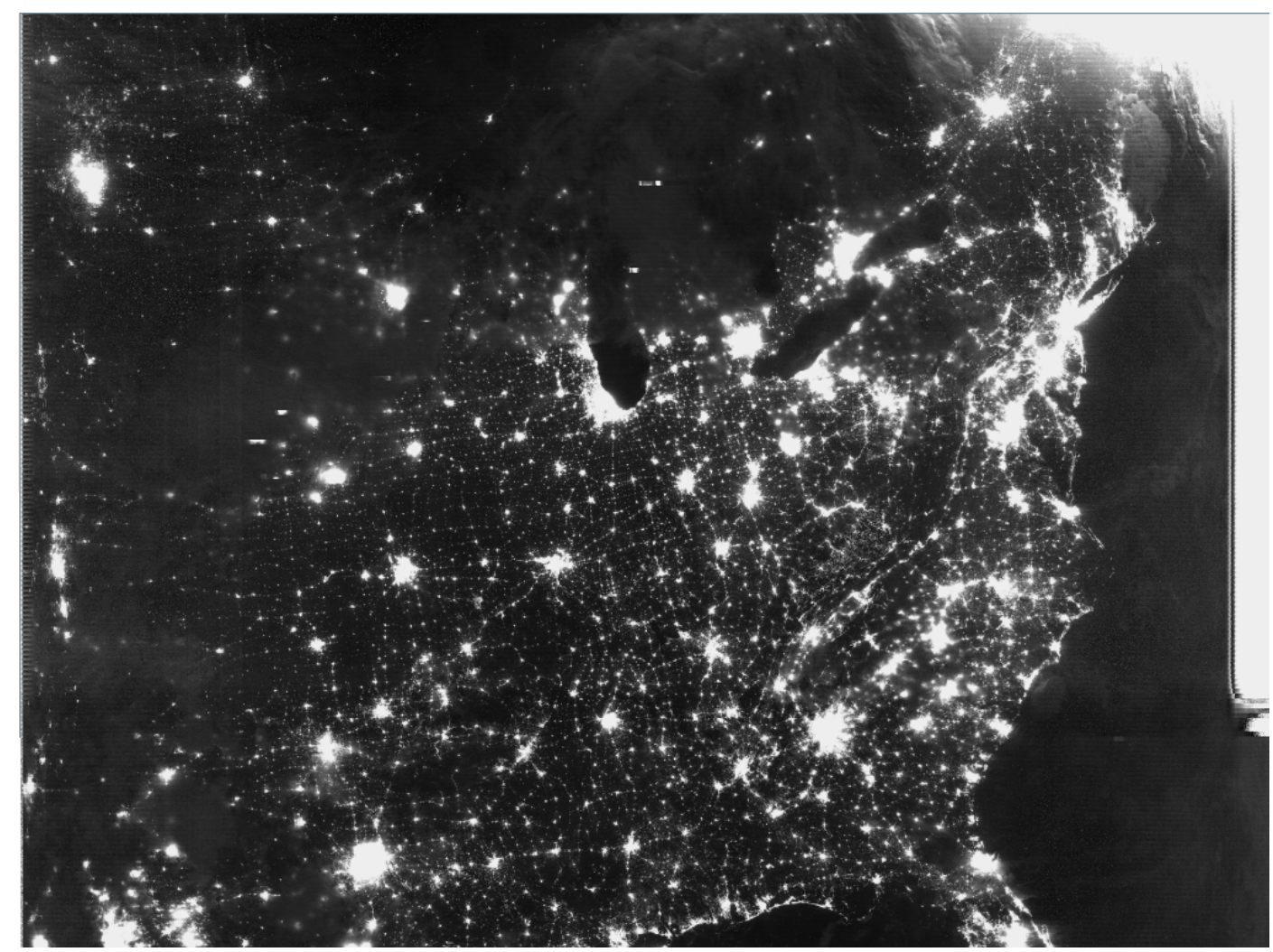

Figure 5 DNB night time scene collected over North America on July 13, 2018, after straylight correction.

Figure 6 and 7 show an example of straylight contaminated nighttime scene over the Southern hemisphere before and after straylight correction, respectively. This example is collected at 23:36:36pm, July 13, 2018. The un-corrected image shows obvious straylight contamination for lower part of the image. There is a clear boundary line between two tunes of straylight contamination as stated previously. Similar to the Northern hemisphere example in Figure 5, after correction, most of straylight contamination is removed but the one at the edge of scan area, where the method did not perform well enough. In Figure 7, a dark horizontal strip is noticeable. It is an over-correction caused by the mis-matched straylight signal entering angle from SD between EV dark signal and the corrected scene, which also occurs in S-NPP DNB straylight affects in the Southern hemisphere over years. 


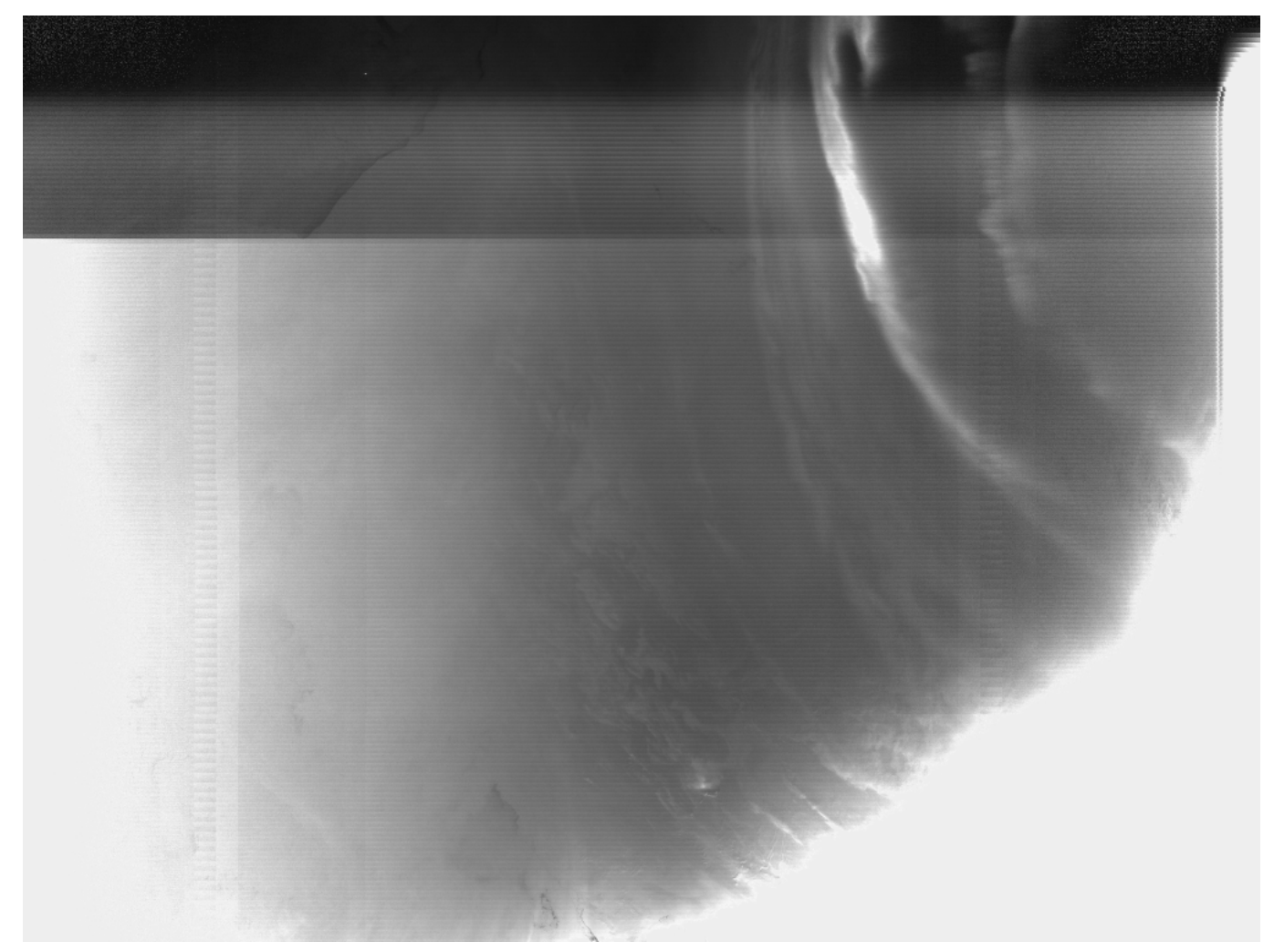

Figure 6 DNB night time scene collected over Southern hemisphere on July 13, 2018, before straylight correction.

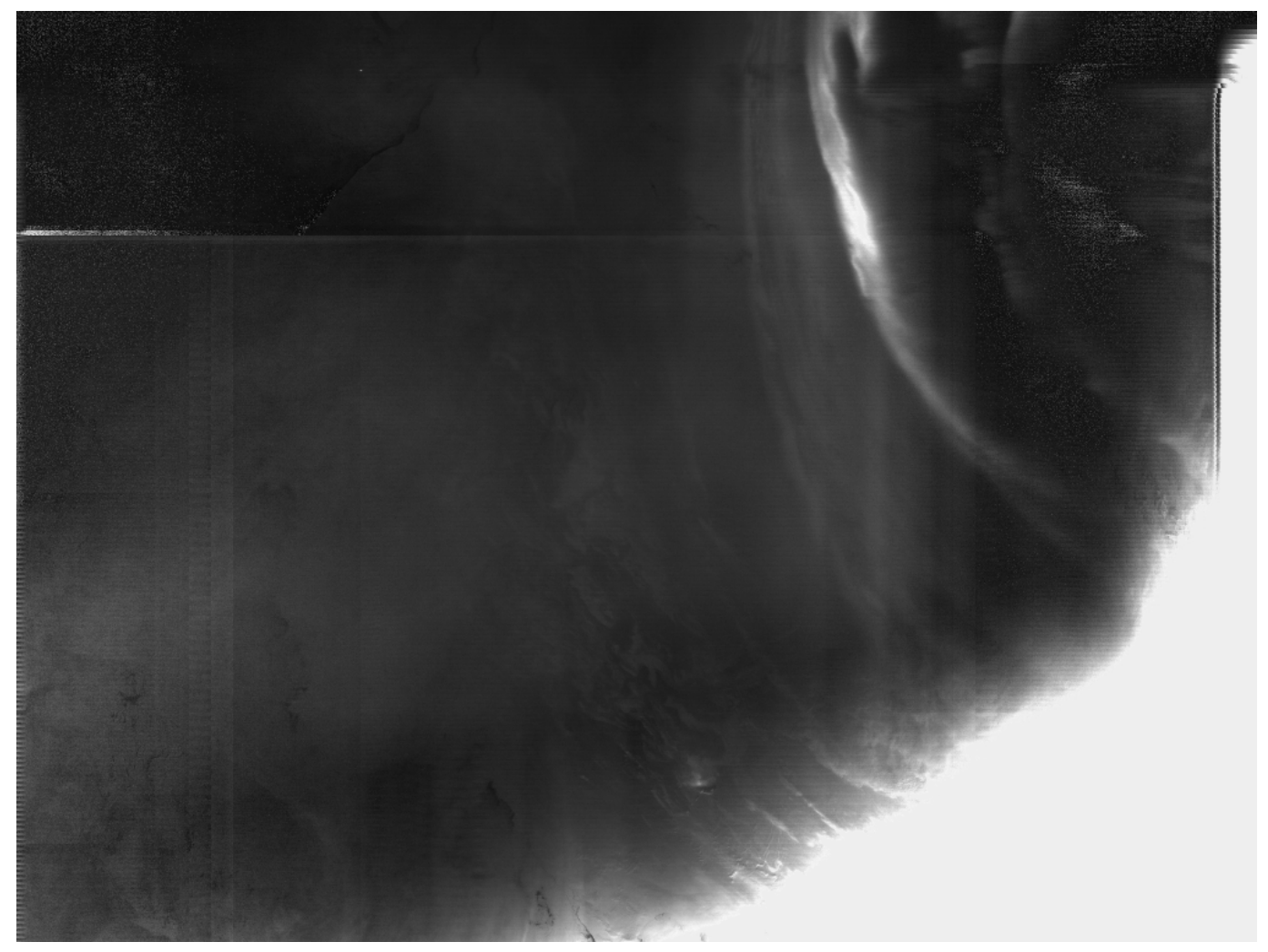

Figure 7 DNB night time scene collected over Southern hemisphere on July 13, 2018, after straylight correction.. 


\section{CONCLUDING REMARKS}

In the early mission, the NOAA-20 VIIRS DNB calibration has been successfully performed. This ultra-sensitive DNB calibration is conducted as a function of aggregation mode, detector, gain stage and HAM-side. The NOAA-20 DNB straylight correction method has been adapted from the S-NPP. In this paper, preliminary results of DNB straylight correction have been illustrated. The straylight contamination magnitudes have been compared between NOAA-20 and S-NPP DNB. Near SD calibration events, DNB stray-light feature is shown to be different in the southern and northern hemispheres. Based on few scenes, The NOAA-20 DNB has much lower straylight contamination, and roughly half to one-third of S-NPP DNB over the same Earth location. The straylight radiance seen by NOAA-20 DNB night image has significantly increased once the cryo-door was open, especially at close to the right edge of scan. Examples of the Northern and Southern hemispheres DNB night images demonstrate the effectiveness of the straylight correction method in major contamination areas. A large contamination in the image right edge of scan area is shown on NOAA-20 night scenes, but not in SNPP. This large straylight feature affects NOAA-20 DNB because it is using a special aggregation mode (Option 21) that extended the EV further than SNPP. Further work needs to be done to remove with this large

straylight contamination if needed. In addition, algorithm refinements will be conducted to improve the straylight characterization to ensure DNB image quality and data products..

\section{ACKNOWLEDGEMENTS}

The authors would like to thank other members of the VCST for their technical discussions and assistance, especially Dr. Kevin Twedt for his helpful comments.

\section{REFERENCES}

[1] S. Millers, S. Weiss and K. Liang, "VIIRS day/night band (DNB) straylight characterization and correction", Proc. SPIE 2013, doi:10.1117/12.2023107.

[2] C. Cao, Y. Bai, “Quantitative Analysis of VIIRS DNB Nightlight Point Source for Light Power Estimation and Stability Monitoring”, Remote Sensing, 2014, 6, 11915-11935.

[3] L. B. Liao, S. Weiss, S. Mills, B. Hauss, "Suomi SNPP VIIRS Day-Night Band On-orbit Performance”, Journal Geophys. Res. Atmos. 2013, 118, 12707-12718.

[4] S. Lee and C. Cao, "Soumi NPP VIIRS Day/Night Band Straylight Characterization and Correction Using Calibration View Data”, Remote Sensing, 2016, 8, 138, February 2016.

[5] S. Lee, K. Chiang, X. Xiong, C. Sun and S. Anderson, "The S-NPP VIIRS Day-Night Band On-orbit

Calibration/Characterization and Current State of SDR Products”, Remote Sensing, 2014, 6, 12427-12446, December 2014.

[6] S. Lee, J. McIntire, H. Oudrari, T. Schwarting, X. Xiong, "A New Method for Suomi-NPP VIIRS Day Night Band (DNB) On-orbit Radiometric Calibration”, IEEE Trans. Geosci. Remote Sensing, 2015, 53, 324-334.

[7] S. Miller, C.L. Combs, S. Q. Kidder and T. Lee, “Assessing Moonlight Availability for Nighttime Environmental Applications by Low-Light Visible Polar-orbiting Satellite Sensors”, Journal of Atmospheric and Oceanic Technology, Vol.29, pp.538-557, April 2012.

[8] R. Andrew, “Kullback-Leibler Approach to Gaussian Mixture Reduction”, IEEE Trans. On Aerospace and Electronic Systems, pp.989-999, vol.43, No.3, July 2007.

[9] T. Cover and J. Thomas, "Elements of Information Theory”, A Wiley-Interscience Publication, John Willey \& Sons, Inc. pp.34, ISBN 0-471-06259-6, Q360.C68, 1991.

[10] John J. Qu, Wei Gao, Menas Kafatos, Robert E. Murphy, Vincent V. Salomonson, "Earth Science Satellite Remote Sensing: Vol.1: Science and Instruments”, Springer, pp.218.

[11] H. Oudrari, J. McIntire, X. Xiong, J. Butler, Q. Ji, T. Schwarting, S. Lee, and B. Efremova, “JPSS-1 VIIRS radiometric characterization and cali- bration based on pre-launch testing,” Remote Sensing 8, 41 (2016). 